\title{
Relationship of serum carotenoid concentrations with allostatic load as a measure of chronic stress among middle-aged adults in the USA
}

\author{
Natalya Rosenberg*, Chang Gi Park and Kamal Eldeirawi \\ Department of Health Systems Science, College of Nursing (M/C 802), University of Illinois at Chicago, \\ 845 South Damen Avenue, Chicago, IL 60612, USA
}

Submitted 22 August 2013: Final revision received 23 December 2013: Accepted 13 January 2014: First published online 11 February 2014

\begin{abstract}
Objective: Chronic stress and repeated physiological attempts at stress adaptation may result in 'fatigue' and suboptimal performance of multiple physiological systems, i.e. allostatic load (AL). Although carotenoids have been linked with individual cardiovascular, metabolic and inflammatory biomarkers, little is known about the relationship of carotenoids with the multi-system biomarker measure of stress, AL. The present study examined the association of serum concentrations of carotenoids with $\mathrm{AL}$ among middle-aged adults.

Design: Cross-sectional. AL score was calculated based on nine risk-rated indicators (systolic and diastolic blood pressure, pulse rate, total and HDL-cholesterol, glycosylated $\mathrm{Hb}$, sex-specific waist-to-hip ratio, albumin and C-reactive protein). Subjects: Middle-aged (45-64 years, $n$ 3387) men and women participants in the Third National Health and Nutrition Examination Survey, NHANES III (1988-1994).

Results: Serum $\beta$-carotene concentration was inversely associated with high $\mathrm{AL}$ after adjusting for age, education, race/ethnicity, serum cotinine, alcohol consumption, physical activity and other carotenoids ( $\alpha$-carotene, $\beta$-cryptoxanthin, lycopene, lutein/zeaxanthin). Females in the lowest $\beta$-carotene quartile were $2 \cdot 94(95 \%$ CI $1 \cdot 74,4 \cdot 94)$ times and males 2.90 (95\% CI $1 \cdot 43,5 \cdot 89)$ times as likely to have high $\mathrm{AL}$, compared with peers in the highest quartile ( $P$ for linear trend 0.001 and 0.018 for females and males, respectively). Mean serum $\beta$-carotene concentrations were also inversely associated with the number of 'high-risk' AL components ( $P$ for linear trend $<0.001$ and 0.004 for females and males, respectively).

Conclusions: Our study adds to evidence linking low $\beta$-carotene levels with unfavourable health outcomes.
\end{abstract}

Keywords
Stress
Physiological
Allostasis
Carotenoids

Chronic stress has been linked with a wide range of health disorders, including metabolic, cardiovascular, mood and cognitive ${ }^{(1-6)}$. Although the exact mechanisms through which chronic stress may lead to negative health outcomes have not been well delineated, according to the allostatic load hypothesis, these pathological effects reflect 'fatigue' of physiological systems from repeating cycles of stress arousal, stress response and post-stress adaptation. The suboptimal performance of multiple physiological systems as a consequence of cumulative stress exposure over a lifetime is termed allostatic load (AL). Biomarkers representing the key physiological players in stress adaptation, namely neuroendocrine, cardiovascular, immune and metabolic systems, have been combined to form composite $\mathrm{AL}$ measures ${ }^{(7-9)}$. These measures have been shown to predict incident physical decline ${ }^{(10)}$, cognitive dysfunction, $\mathrm{CVD}^{(9)}$, frailty $^{(11,12)}$ and mortality ${ }^{(13)}$.

Substantial variability in $\mathrm{AL}$ is evident during the middle age period in adult lifespan. This is the time when $\mathrm{AL}$ reaches its peak and when disparities in AL are especially pronounced ${ }^{(14,15)}$. Therefore, identification of factors associated with AL during middle age may potentially advance our understanding of determinants of stress resilience and vulnerability. Previous studies of $\mathrm{AL}$ have primarily focused on social contextual and individual psychological predictors of $\mathrm{AL}$, such as socio-economic status $^{(16,17)}$, race ${ }^{(15,18,19)}$, neighbourhood characteristics $^{(20,21)}$, social relationships ${ }^{(22,23)}$, coping orientations $^{(24)}$ and stress perception ${ }^{(25)}$. However, there is limited research on the relationship of antioxidant dietary factors with AL. 
Data from animal and human studies indicate that chronic stress is linked to oxidative stress, increased inflammation, and DNA and cellular damage ${ }^{(26-29)}$. Thus, it is plausible that exposure to beneficial antioxidant and anti-inflammatory dietary substances may be associated with variations in $\mathrm{AL}$ vulnerability. Carotenoids are a major class of diet-derived antioxidants with known antiinflammatory, DNA repair, cardio- and cancer-protective properties $^{(30-34)}$. In several cross-sectional and prospective studies, low levels of carotenoids have been linked with certain types of cancer ${ }^{(35)}, \mathrm{CVD}^{(36)}$ and mortality ${ }^{(37)}$. While the association of serum carotenoids with individual cardiovascular, metabolic and inflammatory biomarkers is established in the literature ${ }^{(38-44)}$, little is known about the relationship of serum carotenoids with AL. Identification of nutrients that might contribute to AL will inform the design of prevention strategies to slow down multi-system physiological dysregulation and reduce the impact of chronic stress on morbidity and mortality ${ }^{(45)}$. The purpose of the current study was to examine the association of serum carotenoid concentrations and $\mathrm{AL}$ in a national sample of middle-aged adults (45-64 years) in the USA.

\section{Methods}

\section{Participants}

Data for the current study come from the Third National Health and Nutrition Examination Survey (NHANES III, 1988-1994). The survey is based on a nationally representative sample of the US population, with oversampling of African-Americans, Hispanics and persons aged 60 years and older. Additional information about NHANES III can be found elsewhere ${ }^{(46)}$. The current study included middle-aged adults (45-64 years). Out of 4535 participants (2360 females and 2175 males) who were in the middle age category, 3945 had valid serum carotenoid data and 3914 had valid biological marker data. The final analysis sample was 3387 (1750 females and 1637 males).

\section{Dependent variable}

A composite AL score was created following the method described in a prior AL study ${ }^{(17)}$. The composite score includes cardiovascular (systolic and diastolic blood pressure, pulse rate), metabolic (total cholesterol, HDLcholesterol, glycosylated $\mathrm{Hb}$, sex-specific waist-to-hip ratio) and inflammatory (albumin and C-reactive protein (CRP)) indicators. Each of the nine indicators was dichotomized into 'high $=1$ ' and 'low $=0$ ' risk categories based on clinical criteria. Then, the scores from different indicators were summed to yield a composite AL score (range 0-9). In the composite measure each indicator was equally weighted. Total scores from 0 to 2 were designated as 'low' $\mathrm{AL}$ and scores $\geq 3$ as 'high' AL. The composite score was calculated for participants with complete data on all nine indicators.

Total cholesterol (mg/dl), HDL-cholesterol (mg/dl), CRP $(\mathrm{mg} / \mathrm{dl})$, albumin $(\mathrm{g} / \mathrm{dl})$ and glycosylated $\mathrm{Hb}(\%)$ were measured by contract laboratories using reference analytical methods (for details consult the Laboratory Procedures for NHANES III) ${ }^{(47)}$. Waist-to-hip ratio, radial pulse (beats/min), and systolic and diastolic blood pressure $(\mathrm{mmHg})$ were measured by trained examiners using standard protocols. Blood pressure was measured using a mercury sphygmomanometer (for additional information on these measurements, see NHANES III Examination Data File $\left.{ }^{(48)}\right)$. The arithmetic mean of three systolic and diastolic pressures was used in analysis.

\section{Main independent variables}

The main independent variables examined in the current study included serum levels of $\alpha$-carotene, $\beta$-carotene, $\beta$-cryptoxanthin, lutein/zeaxanthin and lycopene ( $\mu \mathrm{mol} / \mathrm{l})$. These carotenoids were measured in serum by isocratic HPLC, with chromatogram recording at wavelengths of 300, 325 and $450 \mathrm{~nm}$ (for details see Laboratory Procedures for NHANES III) ${ }^{(49)}$.

\section{Covariates}

Covariates examined in the current study included age, educational level, race/ethnicity, smoking status, alcohol consumption and physical activity. Age in years was measured as a continuous variable. Education level was categorized based on the US Census criteria (less than high school, high-school graduate, college graduate). NHANES III race/ethnicity categories included nonHispanic White, non-Hispanic Black, Mexican-American and Other. Smoking status was defined based on serum cotinine levels. Previously reported serum cotinine cut-off points ${ }^{(50)}$ were used to designate non-smokers $(\leq 15 \cdot 0 \mathrm{ng} / \mathrm{ml}(85 \cdot 2 \mathrm{nmol} / \mathrm{l}))$ and smokers $(>15 \cdot 0 \mathrm{ng} / \mathrm{ml}$ $(85 \cdot 2 \mathrm{nmol} / \mathrm{l}))$. Serum cotinine was measured by MS analysis, after an initial screening by the enzyme immunoassay method. Alcohol consumption measure was based on participants' responses about frequency of beer, wine and hard liquor intake in the past month. Summed responses were grouped into alcohol consumption categories 'none', 'moderate' and 'excessive', according to the previously described gender-based criteria $^{(51)}$. For females, moderate alcohol consumption was defined as $1-30$ drinks/month (the equivalent of $\leq 1 \mathrm{drink} / \mathrm{d}$ ). For males, moderate alcohol consumption was defined as 1-60 drinks/month (the equivalent of $\leq 2 \mathrm{drinks} / \mathrm{d}$ ). Physical activity was determined based on the frequency of participation in nine physical activities during the past month. The frequency of each activity was multiplied by its respective metabolic equivalent $(\mathrm{MET})^{(52)}$ to get an intensity-weighted score for each activity. Intensityweighted scores for nine activities (walking, running/ jogging, riding a bicycle, swimming, aerobic dancing, 
other dancing, calisthenics or exercise, gardening/ yardwork, lifting weights) were then summed up and categorized as inactive (values $\leq 3.5$ ) and active (values $>3 \cdot 5)$, as described in prior literature ${ }^{(53)}$.

\section{Statistical analysis}

Analyses were conducted using IBM SPSS Complex Samples version $20 \cdot 0$. All variance estimates were corrected for complex multistage design and population estimates were adjusted based on sample weights provided by NHANES III. $P$ value of $<0.05$ (two-sided) was set as the criterion of significance. The bivariate associations of continuous variables with sex-specific AL were examined using general linear models and the bivariate associations of categorical variables with $\mathrm{AL}$ using $\chi^{2}$ tests. Binary logistic regression was used to model the relationship of individual serum carotenoids ( $\alpha$-carotene, $\beta$-carotene, $\beta$-cryptoxanthin, lutein/zeaxanthin, lycopene) with sex-specific AL, after adjusting for age, education, race/ethnicity, serum cotinine, alcohol consumption, physical activity and other carotenoids. In logistic analysis, serum carotenoid concentrations were categorized into quartiles based on the data in the analysis sample. General linear regression modelling was used to test the linear trends in the relationship between mean concentrations of serum carotenoids and the total AL score, after adjustment for covariates and other carotenoids. In that analysis, the regression of continuous serum concentrations for individual carotenoids $v$. the continuous total AL score was performed. Consistently with prior AL studies ${ }^{(15,19,22,54)}$, we did not control for medication use or doctor-diagnosed chronic conditions as our goal was to capture the existent degree of biological dysfunction.

\section{Results}

A total of 3387 middle-aged participants (1750 females and 1637 males) were included in the study. The mean participant age ranged from 53 to 55 years. The total AL score ranged from 0 to 8 in this sample (maximal possible range $0-9$ ). The mean total AL score was 2.13 and 2.53 for females and males, respectively. The prevalence of high AL was $38.1 \%$ for females and $46 \cdot 8 \%$ for males. Table 1 shows crude associations of AL with covariates. For both sexes, participants with high AL were significantly older $(P<0 \cdot 001)$ and more likely to have lower educational status $(P<0 \cdot 001)$. Race/ethnicity status was significantly associated with high AL among females only $(P<0 \cdot 001)$. Among males, participants with high AL were significantly more likely to be smokers than their male peers with low AL $(P=0 \cdot 011)$. Among females, the proportions of participants who reported moderate as well as excessive alcohol consumption were lower in the high AL group than in the low AL group. The proportion of female nondrinkers was also higher in the high $\operatorname{AL}$ group $(P=0 \cdot 032)$. We also noted a statistically significant inverse association between physical activity status and AL for both females and males $(P<0 \cdot 001$ and $P=0 \cdot 015$, respectively; Table 1$)$.

Table 1 Baseline characteristics by allostatic load (AL) status of middle-aged (45-64 years) participants, Third National Health and Nutrition Examination Survey, NHANES III (1988-1994)

\begin{tabular}{|c|c|c|c|c|c|c|c|c|c|c|}
\hline & \multicolumn{4}{|c|}{ Females } & \multirow[b]{3}{*}{$P+$} & \multicolumn{4}{|c|}{ Males } & \multirow[b]{3}{*}{$P+$} \\
\hline & \multicolumn{2}{|c|}{ Low AL } & \multicolumn{2}{|c|}{ High AL } & & \multicolumn{2}{|c|}{ Low AL } & \multicolumn{2}{|c|}{ High AL } & \\
\hline & $\%^{*}$ & SE & $\%^{*}$ & SE & & $\%^{*}$ & SE & $\%^{*}$ & SE & \\
\hline Prevalence (\%) & $61 \cdot 9$ & $1 \cdot 9$ & $38 \cdot 1$ & $1 \cdot 9$ & $<0.001$ & $53 \cdot 2$ & $2 \cdot 1$ & $46 \cdot 8$ & $2 \cdot 1$ & $<0.001$ \\
\hline \multicolumn{10}{|l|}{ Education (\%) } & $<0.001$ \\
\hline Less than high school & $19 \cdot 1$ & $0 \cdot 3$ & $31 \cdot 0$ & $2 \cdot 4$ & $<0.001$ & $23 \cdot 3$ & $2 \cdot 1$ & $32 \cdot 9$ & $2 \cdot 5$ & $<0.001$ \\
\hline High-school graduate & $62 \cdot 4$ & $1 \cdot 9$ & $56 \cdot 8$ & $2 \cdot 4$ & & $44 \cdot 1$ & $2 \cdot 8$ & $47 \cdot 8$ & $2 \cdot 9$ & \\
\hline College graduate & $18 \cdot 5$ & $1 \cdot 8$ & $12 \cdot 2$ & $1 \cdot 4$ & & $32 \cdot 6$ & $3 \cdot 0$ & $19 \cdot 3$ & $2 \cdot 3$ & \\
\hline \multicolumn{11}{|l|}{ Race/ethnicity (\%) } \\
\hline Non-Hispanic White & $84 \cdot 6$ & $1 \cdot 7$ & $70 \cdot 8$ & $2 \cdot 3$ & $<0.001$ & $82 \cdot 4$ & $1 \cdot 9$ & $79 \cdot 6$ & $2 \cdot 3$ & 0.351 \\
\hline Non-Hispanic Black & $7 \cdot 4$ & $0 \cdot 7$ & $14 \cdot 4$ & $1 \cdot 4$ & & $6 \cdot 3$ & $0 \cdot 7$ & $9 \cdot 1$ & 0.9 & \\
\hline Mexican-American & $2 \cdot 8$ & $0 \cdot 4$ & $5 \cdot 4$ & 0.5 & & $3 \cdot 9$ & 0.5 & 3.9 & 0.5 & \\
\hline Other & $5 \cdot 3$ & $1 \cdot 3$ & $9 \cdot 3$ & $2 \cdot 0$ & & $7 \cdot 4$ & $1 \cdot 6$ & $7 \cdot 4$ & $2 \cdot 2$ & \\
\hline \multicolumn{11}{|l|}{ Smoking status (\%) } \\
\hline Non-smoker & $77 \cdot 0$ & $1 \cdot 9$ & $72 \cdot 6$ & $1 \cdot 8$ & $0 \cdot 071$ & $67 \cdot 1$ & $2 \cdot 6$ & $58 \cdot 0$ & $2 \cdot 9$ & 0.011 \\
\hline Smoker & $23 \cdot 0$ & $1 \cdot 9$ & $27 \cdot 4$ & $1 \cdot 8$ & & $32 \cdot 9$ & $2 \cdot 6$ & $42 \cdot 0$ & $2 \cdot 9$ & \\
\hline \multicolumn{11}{|l|}{ Alcohol consumption (\%) } \\
\hline None & $52 \cdot 9$ & $2 \cdot 8$ & $61 \cdot 9$ & $3 \cdot 2$ & 0.032 & $36 \cdot 7$ & $2 \cdot 6$ & $42 \cdot 7$ & $2 \cdot 4$ & 0.059 \\
\hline Moderate & $42 \cdot 7$ & $2 \cdot 8$ & $35 \cdot 9$ & $3 \cdot 2$ & & $59 \cdot 8$ & $2 \cdot 6$ & $51 \cdot 9$ & $2 \cdot 5$ & \\
\hline Excessive & $4 \cdot 5$ & $1 \cdot 2$ & $2 \cdot 2$ & $0 \cdot 8$ & & 3.5 & $0 \cdot 8$ & $5 \cdot 4$ & $1 \cdot 4$ & \\
\hline \multicolumn{11}{|l|}{ Physical activity (\%) } \\
\hline Inactive & $17 \cdot 0$ & $1 \cdot 7$ & $27 \cdot 9$ & $2 \cdot 0$ & $<0.001$ & $11 \cdot 1$ & $1 \cdot 7$ & $17 \cdot 9$ & $2 \cdot 0$ & 0.015 \\
\hline Active & $83 \cdot 0$ & $1 \cdot 7$ & $72 \cdot 1$ & $2 \cdot 0$ & & 88.9 & $1 \cdot 7$ & $82 \cdot 1$ & $2 \cdot 0$ & \\
\hline
\end{tabular}

${ }^{*}$ Data are weighted percentages, except for Age expressed as mean (in years).

$t P$ values computed using complex-samples general linear model for continuous variables and $\chi^{2}$ test of independence for categorical variables. 
The unadjusted proportions of female and male participants at high risk for individual AL biomarkers are shown in Fig. 1. For both sexes, the leading risk factor was waist-to-hip ratio. This was also the only $\mathrm{AL}$ component for which there were more at-high-risk individuals than at-low-risk individuals $(66 \%$ of females and $92 \%$ of males). The second leading risk factor for

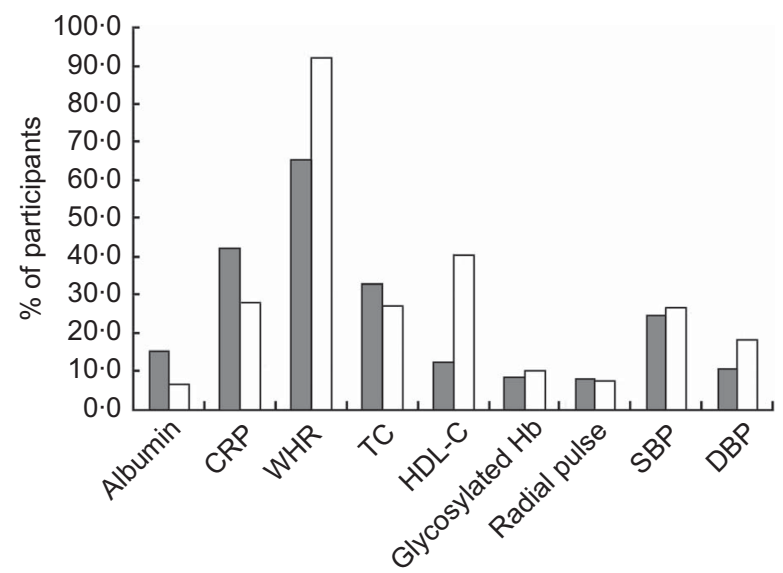

Fig. 1 Unadjusted proportions of middle-aged (45-64 years) participants ( $\square$, females; $\square$, males) at high risk for individual biomarkers of allostatic load from the Third National Health and Nutrition Examination Survey, NHANES III (1988-1994) (CRP, C-reactive protein; WHR, waist-to-hip ratio; TC, total cholesterol; HDL-C, HDL-cholesterol; SBP, systolic blood pressure; DBP, diastolic blood pressure) females was CRP ( $42 \%$ at high risk) and for males, HDL-cholesterol ( $41 \%$ at high risk).

In unadjusted logistic regression analysis, we observed significant dose-response inverse associations of serum concentrations of $\alpha$-carotene, $\beta$-carotene and $\beta$-cryptoxanthin with high $\mathrm{AL}$ in females and males (see Crude model in Tables 2 and 3). These significant trends persisted after adjusting for age, education, race/ ethnicity, serum cotinine, alcohol consumption and physical activity (Partially adjusted model in Tables 2 and 3). However, when we included other carotenoids in the model, only serum concentration of $\beta$-carotene remained inversely and significantly associated with high $\mathrm{AL}$ for both sexes (Fully adjusted model in Tables 2 and 3). In the fully adjusted model, females and males in the lowest quartiles of $\beta$-carotene had a 2.94- and 2.90-fold increased odds of high $\mathrm{AL}$, respectively, compared with their peers in the highest quartiles (95\% CI 1.74, 4.94 and $95 \%$ CI $1 \cdot 43,5 \cdot 89$, respectively). After full adjustment, the linear trend for the association of serum $\beta$-carotene quartiles and high AL also remained significant for both sexes ( $P$ for trend $=0.001$ and 0.018 for females and males, respectively).

In the fully adjusted model, females in the lowest quartile for $\alpha$-carotene had a 1.70 -fold increased odds of high AL (95\% CI 1·04, 2.79, $P$ for trend $=0 \cdot 107$ ), compared with females in the highest quartile. Females in the lowest quartile of lycopene had a $0 \cdot 64$-fold reduced odds of high $\mathrm{AL}(95 \%$ CI $0 \cdot 43,0 \cdot 96, P$ for trend $=0 \cdot 200)$, compared with females in the highest quartile.

Table 2 Logistic regression odds ratios with $95 \%$ confidence intervals of high allostatic load by individual serum carotenoid quartiles in middle-aged (45-64 years) female participants, Third National Health and Nutrition Examination Survey, NHANES III (1988-1994)

\begin{tabular}{|c|c|c|c|c|c|c|c|c|}
\hline & \multicolumn{2}{|c|}{ Quartile 1} & \multicolumn{2}{|c|}{ Quartile 2} & \multicolumn{2}{|c|}{ Quartile 3} & \multirow{2}{*}{$\frac{\text { Quartile } 4}{\text { Ref. }}$} & \multirow[b]{2}{*}{$P$ for linear trenc } \\
\hline & OR & $95 \% \mathrm{Cl}$ & OR & $95 \% \mathrm{Cl}$ & OR & $95 \% \mathrm{Cl}$ & & \\
\hline \multicolumn{9}{|l|}{$\alpha$-Carotene ${ }^{*}$} \\
\hline Crude & $2 \cdot 96$ & $2 \cdot 05,4 \cdot 28$ & $2 \cdot 14$ & $1 \cdot 38,3 \cdot 30$ & $1 \cdot 89$ & $1 \cdot 43,2 \cdot 52$ & 1.00 & $<0.001$ \\
\hline Partially adjustedt & 3.04 & $2 \cdot 09,4 \cdot 42$ & $2 \cdot 30$ & $1 \cdot 46,3 \cdot 62$ & $2 \cdot 04$ & $1 \cdot 49,2 \cdot 81$ & $1 \cdot 00$ & $<0.001$ \\
\hline Fully adjusted $\ddagger$ & $1 \cdot 70$ & $1 \cdot 04,2 \cdot 79$ & $1 \cdot 64$ & $0.98,2 \cdot 73$ & $1 \cdot 65$ & $1 \cdot 09,2 \cdot 49$ & 1.00 & $0 \cdot 107$ \\
\hline \multicolumn{9}{|l|}{$\beta$-Carotene ${ }^{\star}$} \\
\hline Crude & $3 \cdot 47$ & $2 \cdot 38,5 \cdot 07$ & $2 \cdot 21$ & $1 \cdot 36,3 \cdot 57$ & $1 \cdot 52$ & $1 \cdot 08,2 \cdot 13$ & 1.00 & $<0.001$ \\
\hline Partially adjusted & $3 \cdot 50$ & $2 \cdot 32,5 \cdot 26$ & $2 \cdot 40$ & $1 \cdot 46,3 \cdot 94$ & $1 \cdot 50$ & $1 \cdot 06,2 \cdot 12$ & 1.00 & $<0.001$ \\
\hline Fully adjusted & $2 \cdot 94$ & $1 \cdot 74,4.94$ & $2 \cdot 05$ & $1 \cdot 09,3 \cdot 83$ & $1 \cdot 37$ & $0 \cdot 88,2 \cdot 11$ & $1 \cdot 00$ & 0.001 \\
\hline \multicolumn{9}{|l|}{$\beta$-Cryptoxanthin* } \\
\hline Crude & $1 \cdot 85$ & $1 \cdot 20,2 \cdot 86$ & $1 \cdot 12$ & $0.77,1.63$ & 0.99 & $0.68,1.43$ & $1 \cdot 00$ & 0.032 \\
\hline Partially adjusted & 1.99 & $1 \cdot 26,3 \cdot 15$ & $1 \cdot 26$ & $0.87,1 \cdot 81$ & 1.09 & $0.78,1.52$ & 1.00 & 0.043 \\
\hline Fully adjusted & $1 \cdot 10$ & $0 \cdot 60,2 \cdot 00$ & $0 \cdot 86$ & $0.52,1.41$ & $0 \cdot 86$ & $0.58,1 \cdot 26$ & 1.00 & $0 \cdot 702$ \\
\hline \multicolumn{9}{|l|}{ Lutein/zeaxanthin* } \\
\hline Crude & $1 \cdot 21$ & $0 \cdot 72,2 \cdot 04$ & $1 \cdot 02$ & $0.68,1.54$ & $0 \cdot 86$ & $0.57,1.29$ & 1.00 & $0 \cdot 391$ \\
\hline Partially adjusted & $1 \cdot 34$ & $0 \cdot 75,2 \cdot 38$ & $1 \cdot 09$ & $0.71,1.67$ & 0.87 & $0.55,1.35$ & $1 \cdot 00$ & 0.318 \\
\hline Fully adjusted & $0 \cdot 76$ & $0.39,1.47$ & $0 \cdot 78$ & $0 \cdot 49,1 \cdot 24$ & 0.77 & $0.51,1 \cdot 16$ & 1.00 & 0.630 \\
\hline \multicolumn{9}{|l|}{ Lycopene ${ }^{\star}$} \\
\hline Crude & $1 \cdot 34$ & $0 \cdot 95,1 \cdot 89$ & $1 \cdot 17$ & $0 \cdot 80,1 \cdot 72$ & 0.93 & $0 \cdot 65,1 \cdot 34$ & $1 \cdot 00$ & 0.238 \\
\hline Partially adjusted & $1 \cdot 00$ & $0 \cdot 70,1 \cdot 44$ & $1 \cdot 06$ & $0.73,1.55$ & 0.97 & $0 \cdot 67,1 \cdot 38$ & 1.00 & 0.959 \\
\hline Fully adjusted & 0.64 & $0.43,0.96$ & 0.79 & $0.51,1.22$ & 0.85 & $0.58,1.24$ & $1 \cdot 00$ & 0.200 \\
\hline
\end{tabular}

Ref., referent category.

${ }^{*} \mu \mathrm{mol} / \mathrm{l}$; to convert from $\mu \mathrm{mol} / \mathrm{l}$ to $\mu \mathrm{g} / \mathrm{l}$ units divide $\alpha$-carotene, $\beta$-carotene and lycopene serum concentrations by 0.001863 ; $\beta$-cryptoxanthin serum concentrations by 0.001809 ; and lutein/zeaxanthin serum concentrations by 0.001758 .

tAdjusted for age, education, race/ethnicity, serum cotinine, alcohol consumption and physical activity.

$\ddagger$ Adjusted for age, education, race/ethnicity, serum cotinine, alcohol consumption, physical activity and other carotenoids. 
Table 3 Logistic regression odds ratios with $95 \%$ confidence intervals of high allostatic load by individual serum carotenoid quartiles in middle-aged (45-64 years) male participants, Third National Health and Nutrition Examination Survey, NHANES III (1988-1994)

\begin{tabular}{|c|c|c|c|c|c|c|c|c|}
\hline & \multicolumn{2}{|c|}{ Quartile 1} & \multicolumn{2}{|c|}{ Quartile 2} & \multicolumn{2}{|c|}{ Quartile 3} & \multirow{2}{*}{$\frac{\text { Quartile } 4}{\text { Ref. }}$} & \multirow[b]{2}{*}{$P$ for linear trend } \\
\hline & OR & $95 \% \mathrm{Cl}$ & OR & $95 \% \mathrm{Cl}$ & OR & $95 \% \mathrm{Cl}$ & & \\
\hline \multicolumn{9}{|l|}{$\alpha$-Carotene* } \\
\hline Crude & $3 \cdot 32$ & $2 \cdot 07,5 \cdot 32$ & 1.58 & $1 \cdot 04,2 \cdot 40$ & $1 \cdot 39$ & $0.93,2.09$ & $1 \cdot 00$ & $<0.001$ \\
\hline Partially adjustedt & $3 \cdot 05$ & $1 \cdot 72,5 \cdot 41$ & $1 \cdot 52$ & $0.96,2 \cdot 42$ & $1 \cdot 38$ & $0.92,2.06$ & $1 \cdot 00$ & 0.002 \\
\hline Fully adjustedł & $1 \cdot 63$ & $0.80,3.32$ & 0.92 & $0.54,1.59$ & 0.94 & $0.61,1.45$ & $1 \cdot 00$ & 0.081 \\
\hline \multicolumn{9}{|l|}{$\beta$-Carotene* } \\
\hline Crude & $3 \cdot 14$ & $1 \cdot 83,5 \cdot 37$ & $2 \cdot 33$ & $1 \cdot 47,3 \cdot 69$ & $1 \cdot 60$ & $0.93,2 \cdot 77$ & $1 \cdot 00$ & $<0.001$ \\
\hline Partially adjusted & $3 \cdot 48$ & $1 \cdot 90,6 \cdot 37$ & $2 \cdot 50$ & $1 \cdot 46,4 \cdot 29$ & $1 \cdot 80$ & $1 \cdot 01,3 \cdot 20$ & $1 \cdot 00$ & 0.001 \\
\hline Fully adjusted & $2 \cdot 90$ & $1 \cdot 43,5 \cdot 89$ & $2 \cdot 53$ & $1 \cdot 39,4.59$ & $1 \cdot 78$ & $0.96,3.31$ & 1.00 & 0.018 \\
\hline \multicolumn{9}{|l|}{$\beta$-Cryptoxanthin* } \\
\hline Crude & $2 \cdot 57$ & $1 \cdot 68,3.94$ & $1 \cdot 44$ & $0 \cdot 96,2 \cdot 16$ & $1 \cdot 02$ & $0.62,1.68$ & $1 \cdot 00$ & $<0.001$ \\
\hline Partially adjusted & $2 \cdot 18$ & $1 \cdot 29,3.68$ & $1 \cdot 45$ & $0 \cdot 88,2 \cdot 39$ & 0.96 & $0.55,1.69$ & 1.00 & 0.01 \\
\hline Fully adjusted & $1 \cdot 39$ & $0.74,2 \cdot 60$ & $1 \cdot 11$ & $0.65,1 \cdot 87$ & 0.82 & $0 \cdot 45,1 \cdot 47$ & $1 \cdot 00$ & $0 \cdot 284$ \\
\hline \multicolumn{9}{|l|}{ Lutein/zeaxanthin* } \\
\hline Crude & 1.55 & $1 \cdot 00,2 \cdot 41$ & 1.09 & $0 \cdot 70,1 \cdot 71$ & $1 \cdot 40$ & $0 \cdot 90,2 \cdot 17$ & $1 \cdot 00$ & $0 \cdot 152$ \\
\hline Partially adjusted & $1 \cdot 37$ & $0 \cdot 89,2 \cdot 12$ & 0.96 & $0.59,1.56$ & $1 \cdot 33$ & $0.84,2.09$ & $1 \cdot 00$ & $0 \cdot 15$ \\
\hline Fully adjusted & $0 \cdot 76$ & $0 \cdot 48,1 \cdot 21$ & 0.68 & $0.43,1.06$ & $1 \cdot 12$ & $0.72,1.75$ & $1 \cdot 00$ & 0.095 \\
\hline \multicolumn{9}{|l|}{ Lycopene* } \\
\hline Crude & 1.59 & $1 \cdot 08,2 \cdot 33$ & $1 \cdot 27$ & $0.83,1.93$ & $1 \cdot 16$ & $0.79,1.69$ & $1 \cdot 00$ & $0 \cdot 124$ \\
\hline Partially adjusted & $1 \cdot 16$ & $0 \cdot 77,1 \cdot 74$ & $1 \cdot 10$ & $0 \cdot 70,1 \cdot 71$ & $1 \cdot 08$ & $0.74,1.59$ & $1 \cdot 00$ & 0.91 \\
\hline Fully adjusted & 0.99 & $0.43,1 \cdot 11$ & 0.91 & $0.60,1.39$ & 0.89 & $0.62,1 \cdot 27$ & $1 \cdot 00$ & 0.483 \\
\hline
\end{tabular}

Ref., referent category.

${ }^{*} \mu \mathrm{mol} / /$; to convert from $\mu \mathrm{mol} / \mathrm{l}$ to $\mu \mathrm{g} / \mathrm{l}$ units divide $\alpha$-carotene, $\beta$-carotene and lycopene serum concentrations by $0 \cdot 001863 ; \beta$-cryptoxanthin serum concentrations by 0.001809 ; and lutein/zeaxanthin serum concentrations by 0.001758 .

tAdjusted for age, education, race/ethnicity, serum cotinine, alcohol consumption and physical activity.

$\ddagger$ Adjusted for age, education, race/ethnicity, serum cotinine, alcohol consumption, physical activity and other carotenoids.

Table 4 Mean concentrations* of serum carotenoids by the number of 'high-risk' allostatic load (AL) componentst, adjustedł for covariates, in middle-aged (45-64 years) participants, Third National Health and Nutrition Examination Survey, NHANES III (1988-1994)

\begin{tabular}{|c|c|c|c|c|c|c|c|c|c|c|c|c|c|}
\hline & \multicolumn{12}{|c|}{ Number of AL components } & \multirow[b]{3}{*}{$P$ for linear trend } \\
\hline & \multicolumn{2}{|c|}{0} & \multicolumn{2}{|c|}{1} & \multicolumn{2}{|c|}{2} & \multicolumn{2}{|c|}{3} & \multicolumn{2}{|c|}{4} & \multicolumn{2}{|c|}{$\geq 5$} & \\
\hline & Mean & $\mathrm{SE}$ & Mean & SE & Mean & SE & Mean & SE & Mean & SE & Mean & SE & \\
\hline \multicolumn{14}{|l|}{$\alpha$-Carotene } \\
\hline Females & $0 \cdot 12$ & 0.01 & $0 \cdot 10$ & 0.01 & $0 \cdot 11$ & 0.01 & $0 \cdot 10$ & 0.01 & $0 \cdot 11$ & 0.01 & $0 \cdot 11$ & 0.01 & 0.581 \\
\hline Males & 0.07 & 0.01 & 0.09 & 0.01 & 0.08 & 0.01 & 0.09 & 0.01 & 0.08 & 0.01 & 0.08 & 0.01 & $0 \cdot 155$ \\
\hline \multicolumn{14}{|l|}{$\beta$-Carotene } \\
\hline Females & 0.49 & 0.03 & 0.46 & 0.02 & 0.44 & 0.03 & $0 \cdot 39$ & 0.03 & 0.38 & 0.03 & 0.34 & 0.03 & $<0.001$ \\
\hline Males & 0.36 & 0.03 & 0.33 & 0.03 & 0.35 & 0.02 & $0 \cdot 28$ & 0.02 & 0.29 & 0.02 & 0.30 & 0.03 & 0.004 \\
\hline \multicolumn{14}{|c|}{$\beta$-Cryptoxanthin } \\
\hline Females & 0.22 & 0.01 & $0 \cdot 21$ & 0.01 & $0 \cdot 20$ & 0.01 & $0 \cdot 20$ & 0.01 & $0 \cdot 19$ & 0.01 & $0 \cdot 20$ & 0.01 & $0 \cdot 128$ \\
\hline Males & $0 \cdot 18$ & 0.02 & $0 \cdot 17$ & 0.01 & $0 \cdot 17$ & 0.01 & $0 \cdot 17$ & 0.01 & $0 \cdot 16$ & 0.01 & $0 \cdot 17$ & 0.01 & 0.324 \\
\hline \multicolumn{14}{|c|}{ Lutein/zeaxanthin } \\
\hline Females & 0.47 & 0.02 & 0.45 & 0.02 & 0.45 & 0.02 & 0.45 & 0.02 & 0.47 & 0.02 & 0.45 & 0.02 & 0.788 \\
\hline Males & 0.46 & 0.04 & 0.44 & 0.02 & 0.43 & 0.02 & 0.43 & 0.02 & 0.46 & 0.02 & 0.47 & 0.03 & 0.403 \\
\hline \multicolumn{14}{|l|}{ Lycopene } \\
\hline Females & 0.30 & 0.02 & 0.33 & 0.01 & 0.38 & 0.02 & 0.39 & 0.02 & $0 \cdot 36$ & 0.02 & 0.37 & 0.03 & 0.012 \\
\hline Males & 0.40 & 0.03 & 0.35 & 0.02 & 0.37 & 0.02 & $0 \cdot 37$ & 0.01 & 0.35 & 0.02 & 0.39 & 0.03 & $0 \cdot 707$ \\
\hline
\end{tabular}

${ }^{*} \mu \mathrm{mol} / \mathrm{l}$; to convert from $\mu \mathrm{mol} / \mathrm{l}$ to $\mu \mathrm{g} / \mathrm{l}$ units divide $\alpha$-carotene, $\beta$-carotene and lycopene serum concentrations by 0.001863 ; $\beta$-cryptoxanthin serum concentrations by 0.001809 ; and lutein/zeaxanthin serum concentrations by 0.001758 .

tThe number of AL components in the high risk category, possible score range 0-9, sample score range 0-8; score categories 5-8 were combined because of low cell size.

$\ddagger$ Adjusted for age, education, race/ethnicity, serum cotinine, alcohol consumption, physical activity and other carotenoids.

To rule out potential confounding of the serum carotenoids-AL relationship from the ongoing acute inflammatory processes, we repeated analyses after excluding participants with white blood cell count greater than 10000 cells $/ \mu$ l and those who responded 'Yes' to a survey question 'In the past few days have you had a cough, cold, or other acute illness?' (excluded females $n$ 542, males $n$ 499). After exclusion, the fully adjusted odds of high AL for all carotenoids did not change appreciably for both sexes (results not shown).

To test whether individual serum carotenoid concentrations were associated with the total number of 
'high-risk' AL components, i.e. total AL score, we performed a multiple linear regression analysis. For females, serum $\beta$-carotene concentration was inversely associated with the total AL score $(P$ for trend $<0 \cdot 001)$. Compared with females with a total AL score of 0 , females with a total AL score $\geq 5$ had a $31 \%$ lower mean $\beta$-carotene concentration. For males, serum $\beta$-carotene concentration was inversely associated with the total AL score ( $P$ for trend $=0.004)$. Compared with males with a total $\mathrm{AL}$ score of 0 , males with a total AL score of $\geq 5$ had a $17 \%$ lower mean $\beta$-carotene concentration (Table 4).

\section{Discussion}

To our knowledge, the current study is the first one to examine the association of serum carotenoid concentrations with $\mathrm{AL}$ in a representative sample of the US middle-aged population. We found significant dose-response, inverse associations of serum $\beta$-carotene concentrations and high AL risk in females and males. These trends persisted after we accounted for age, education, race, smoking, alcohol consumption and physical activity, as well as evidence of ongoing or recent acute inflammation. Our results also demonstrated that mean serum $\beta$-carotene concentrations decreased in a linear fashion with increasing number of high-risk AL biomarker components.

The construct of AL represents a composite biomarker signature of multi-system 'wear and tear' from cumulative chronic stress exposure over a lifetime. Our findings, thus, may signify that $\beta$-carotene has broad biological significance across multiple physiological systems. It is likely that $\beta$-carotene supports optimal physiological performance though its various bioregulatory functions, including genetic modulation of oxidative stress pathways $^{(55,56)}$, upregulation of immune response ${ }^{(31)}$ and pro-vitamin A activity ${ }^{(33,57)}$. Alternatively, depletion of serum $\beta$-carotene levels may result in the setting of adverse physiological conditions, e.g. conditions of increased oxidative stress and inflammation ${ }^{(38,58-60)}$. Our findings are consistent with the results of other studies showing an inverse association of serum $\beta$-carotene with abnormalities in individual AL biomarkers, including abdominal obesity ${ }^{(40)}, \mathrm{BMI}^{(38)}$, insulin resistance ${ }^{(44)}$, hypertension $^{(40)}$ and $\mathrm{CRP}^{(39,41,43)}$.

Biological interrelationships among carotenoids and various dietary antioxidants have been increasingly emphasized in the literature ${ }^{(37,61-63)}$. That is why all our analyses were adjusted for other individual carotenoids. Our results clearly demonstrate that carotenoids have a modulating influence on each other. For example, the association of $\alpha$-carotene and $\beta$-cryptoxanthin with $\mathrm{AL}$, found to be significant in the crude and partially adjusted analyses, ceased to be significant after adjustment for other carotenoids. In contrast, the inverse association of $\beta$-carotene with $\mathrm{AL}$ remained significant for both sexes even after adjustment for other carotenoids. This finding suggests that serum levels of $\beta$-carotene are more strongly determined by the physiological milieu than by the presence of other carotenoids. Additional research on physiological interaction of various carotenoids is warranted.

A noteworthy observation in the current study is that a disproportionately higher number of participants in this national sample of middle-aged adults were in the high risk category for central obesity and CRP than for any other component of AL. Weight gain has been characterized in the theoretical literature as a natural biological mechanism for adaption to environmental demands ${ }^{(64,65)}$. Empirical data linking stress and obesity $^{(66-68)}$ and epidemiological trends indicating that onset of obesity occurs earlier in modern US cohorts ${ }^{(69)}$ indeed suggest that metabolic mechanisms of stress adaptation may be predominant in the years leading to middle age. The role of obesity/inflammation in the development of $\mathrm{AL}$ across the lifespan needs to be examined in future longitudinal studies. Individual AL biomarker components, currently receiving equal weight in the composite AL measures, may need to be weighted differentially based on the timing and duration of risk factor exposure. Additionally, given the characteristics of the sample, it is possible that the observed inverse association of serum $\beta$-carotene with AL was heavily influenced by its known strong association with obesity ${ }^{(38,42)}$ and $\mathrm{CRP}^{(39,43)}$. Previous studies suggest that the inflammatory environment ${ }^{(58-60,70,71)}$ and obesity-linked oxidation of the lipoprotein carriers of $\beta$-carotene, specifically $\mathrm{LDL}^{(72,73)}$, may be related to low serum $\beta$-carotene levels.

The risk of high AL has been linked with lower educational status $^{(17)}$, minority racial status ${ }^{(15)}$, low physical activity $^{(54,74,75)}$ and smoking ${ }^{(76)}$ in previous investigations. These associations have been confirmed in our study. In our crude analysis, we also noted that females with high AL were less likely to report drinking alcohol compared with their female counterparts with low AL. Although a similar association between reduced alcohol consumption and high AL has been observed in a study of middle-age and older Puerto Rican adults ${ }^{(54)}$, these are bivariate results and should be considered with caution. Considerable methodological differences across studies that examined the association of alcohol consumption with $\mathrm{AL}^{(54,75,76)}$ currently prevent drawing any conclusions about this relationship.

Generally, the association trends between serum carotenoids and AL were similar for both sexes. Two sex-based differences in the results are worth noting, however. First, the linear trends for the inverse association of serum $\beta$-carotene quartiles and high $\mathrm{AL}$, and of mean serum $\beta$-carotene concentrations with the number of high-risk AL components, were more pronounced in females than in males. It is possible that gender differences in $\mathrm{AL}$ profile are responsible for these dissimilarities. As has 
been pointed out earlier, males in this sample were more likely to be centrally obese and have low HDL-cholesterol, while females were more likely to be centrally obese and have elevated CRP. Second, the lowest quartile of serum $\alpha$-carotene was significantly associated with high AL risk in females only. Since female populations tend to have higher $\alpha$ - and $\beta$-carotene levels than male populations ${ }^{(77,78)}$, it is possible that lower serum $\alpha$ - and $\beta$-carotene status is a more sensitive indicator of increasing physiological dysfunction in females than in males.

Our findings should be interpreted with caution. First, causality of the association between serum carotenoids and AL cannot be assumed based on the cross-sectional design of the study; however, our results point to an increased vulnerability to high AL in individuals with low serum $\beta$-carotene levels. Second, theoretical refinement of the AL construct is still needed to aid interpretations of AL studies. For example, the cut-off point for designating high AL was based on the example of previous AL studies. No strong theoretical justification for this cut-off point exists, however.

Our study has several strengths. First, our study contributes to a still limited research on the relationship of antioxidant status and the measure of cumulative stress exposure, AL. Second, the study utilized a nationally representative sample of middle-aged adults, which enhances the generalizability of our findings. Third, we examined the middle age population, which exhibits significant variation in AL levels yet has received relatively less attention in AL studies.

\section{Conclusion}

Our results demonstrate a strong inverse association of serum $\beta$-carotene with the measure of cumulative stress exposure, AL. Further research is needed to determine whether chronic stress is the mechanism linking low levels of serum $\beta$-carotene with adverse health outcomes.

\section{Acknowledgements}

Sources of funding: The work of K.E. on this manuscript was partially supported by the Robert Wood Johnson Foundation Nurse Faculty Scholar Program (Grant \# 71249). The funder made no contributions to the study design, analysis or interpretation. Conflicts of interest: There are no conflicts of interest, including specific financial interests or affiliations relevant to the subject of this manuscript. Ethics statement: This research has been determined as exempt by the University of Illinois at Chicago Institutional Review Board. Authors' contributions: N.R. designed the study, performed data analysis and wrote the manuscript. C.G.P. assisted with statistical analysis and interpretation of data. K.E. supervised the study design, analysis and interpretation, and made significant contributions to the manuscript preparation. All authors contributed to the development of the manuscript.

\section{References}

1. Bose M, Olivan B \& Laferrere B (2009) Stress and obesity: the role of the hypothalamic-pituitary-adrenal axis in metabolic disease. Curr Opin Endocrinol Diabetes Obes 16, 340-346.

2. Conrad CD \& Bimonte-Nelson HA (2010) Impact of the hypothalamic-pituitary-adrenal/gonadal axes on trajectory of age-related cognitive decline. Prog Brain Res 182, 31-76.

3. Heffner KL (2011) Neuroendocrine effects of stress on immunity in the elderly: implications for inflammatory disease. Immunol Allergy Clin North Am 31, 95-108.

4. Marin MF, Lord C, Andrews J et al. (2011) Chronic stress, cognitive functioning and mental health. Neurobiol Learn Mem 96, 583-595.

5. Steptoe A \& Kivimaki M (2012) Stress and cardiovascular disease. Nat Rev Cardiol 9, 360-370.

6. Tamashiro KL, Sakai RR, Shively CA et al. (2011) Chronic stress, metabolism, and metabolic syndrome. Stress 14, 468-474

7. McEwen BS (2008) Central effects of stress hormones in health and disease: understanding the protective and damaging effects of stress and stress mediators. Eur $J$ Pharmacol 583, 174-185.

8. McEwen BS (1998) Protective and damaging effects of stress mediators. $N$ Engl J Med 338, 171-179.

9. Seeman TE, Singer BH, Rowe JW et al. (1997) Price of adaptation - allostatic load and its health consequences. MacArthur studies of successful aging. Arch Intern Med 157, 2259-2268.

10. Karlamangla AS, Singer BH, McEwen BS et al. (2002) Allostatic load as a predictor of functional decline. MacArthur studies of successful aging. J Clin Epidemiol 55, 696-710.

11. Gruenewald TL, Seeman TE, Karlamangla AS et al. (2009) Allostatic load and frailty in older adults. J Am Geriatr Soc 57, 1525-1531.

12. Szanton SL, Allen JK, Seplaki CL et al. (2009) Allostatic load and frailty in the women's health and aging studies. Biol Res Nurs 10, 248-256.

13. Seeman TE, McEwen BS, Rowe JW et al. (2001) Allostatic load as a marker of cumulative biological risk: MacArthur studies of successful aging. Proc Natl Acad Sci USA 98, 4770-4775.

14. Crimmins EM, Johnston M, Hayward M et al. (2003) Age differences in allostatic load: an index of physiological dysregulation. Exp Gerontol 38, 731-734.

15. Chyu L \& Upchurch DM (2011) Racial and ethnic patterns of allostatic load among adult women in the United States: findings from the National Health and Nutrition Examination Survey 1999-2004. J Womens Health (Larchmt) 20, 575-583.

16. Gruenewald TL, Karlamangla AS, Hu P et al. (2012) History of socioeconomic disadvantage and allostatic load in later life. Soc Sci Med 74, 75-83.

17. Seeman T, Merkin SS, Crimmins E et al. (2008) Education, income and ethnic differences in cumulative biological risk profiles in a national sample of US adults: NHANES III (1988-1994). Soc Sci Med 66, 72-87.

18. Deuster PA, Kim-Dorner SJ, Remaley AT et al. (2011) Allostatic load and health status of African Americans and whites. Am J Health Behav 35, 641-653.

19. Geronimus AT, Hicken M, Keene D et al. (2006) 'Weathering' and age patterns of allostatic load scores among blacks and whites in the United States. Am J Public Health 96, 826-833. 
20. Bird CE, Seeman T, Escarce JJ et al. (2010) Neighbourhood socioeconomic status and biological 'wear and tear' in a nationally representative sample of US adults. $J$ Epidemiol Community Health 64, 860-865.

21. Merkin SS, Basurto-Davila R, Karlamangla A et al. (2009) Neighborhoods and cumulative biological risk profiles by race/ethnicity in a national sample of US adults: NHANES III. Ann Epidemiol 19, 194-201.

22. Seeman TE, Singer BH, Ryff CD et al. (2002) Social relationships, gender, and allostatic load across two age cohorts. Psychosom Med 64, 395-406.

23. Singer B \& Ryff CD (1999) Hierarchies of life histories and associated health risks. Ann N Y Acad Sci 896, 96-115.

24. Chen E, Miller GE, Lachman ME et al. (2012) Protective factors for adults from low-childhood socioeconomic circumstances: the benefits of shift-and-persist for allostatic load. Psychosom Med 74, 178-186.

25. Glei DA, Goldman N, Chuang YL et al. (2007) Do chronic stressors lead to physiological dysregulation? Testing the theory of allostatic load. Psychosom Med 69, 769-776.

26. Bauer ME, Jeckel CM \& Luz C (2009) The role of stress factors during aging of the immune system. Ann $N$ Y Acad Sci 1153, 139-152.

27. Epel ES (2009) Psychological and metabolic stress: a recipe for accelerated cellular aging? Hormones (Athens) 8, 7-22.

28. Hansel A, Hong S, Camara RJ et al. (2010) Inflammation as a psychophysiological biomarker in chronic psychosocial stress. Neurosci Biobehav Rev 35, 115-121.

29. O'Donovan A, Tomiyama AJ, Lin J et al. (2012) Stress appraisals and cellular aging: a key role for anticipatory threat in the relationship between psychological stress and telomere length. Brain Behav Immun 26, 573-579.

30. Agarwal M, Parameswari RP, Vasanthi HR et al. (2012) Dynamic action of carotenoids in cardioprotection and maintenance of cardiac health. Molecules 17, 4755-4769.

31. Chew BP \& Park JS (2004) Carotenoid action on the immune response. J Nutr 134, issue 1, 257S-261S.

32. Lorenzo Y, Azqueta A, Luna L et al. (2009) The carotenoid $\beta$-cryptoxanthin stimulates the repair of DNA oxidation damage in addition to acting as an antioxidant in human cells. Carcinogenesis 30, 308-314.

33. Rao AV \& Rao LG (2007) Carotenoids and human health. Pharmacol Res 55, 207-216.

34. Tapiero H, Townsend DM \& Tew KD (2004) The role of carotenoids in the prevention of human pathologies. Biomed Pharmacother 58, 100-110.

35. Miller PE \& Snyder DC (2012) Phytochemicals and cancer risk: a review of the epidemiological evidence. Nutr Clin Pract 27, 599-612.

36. Willcox BJ, Curb JD \& Rodriguez BL (2008) Antioxidants in cardiovascular health and disease: key lessons from epidemiologic studies. Am J Cardiol 101, 75D-86D.

37. Shardell MD, Alley DE, Hicks GE et al. (2011) Low-serum carotenoid concentrations and carotenoid interactions predict mortality in US adults: the Third National Health and Nutrition Examination Survey. Nutr Res 31, 178-189.

38. Andersen LF, Jacobs DR Jr, Gross MD et al. (2006) Longitudinal associations between body mass index and serum carotenoids: the CARDIA study. Br J Nutr 95, 358-365.

39. Erlinger TP, Guallar E, Miller ER 3rd, et al. (2001) Relationship between systemic markers of inflammation and serum $\beta$-carotene levels. Arch Intern Med 161, 1903-1908.

40. Ford ES, Mokdad AH, Giles WH et al. (2003) The metabolic syndrome and antioxidant concentrations: findings from the Third National Health and Nutrition Examination Survey. Diabetes 52, 2346-2352.

41. Ford ES, Liu S, Mannino DM et al. (2003) C-reactive protein concentration and concentrations of blood vitamins, carotenoids, and selenium among United States adults. Eur J Clin Nutr 57, 1157-1163.
42. Kimmons JE, Blanck HM, Tohill BC et al. (2006) Associations between body mass index and the prevalence of low micronutrient levels among US adults. Med Gen Med 8, 59.

43. Kritchevsky SB, Bush AJ, Pahor M et al. (2000) Serum carotenoids and markers of inflammation in nonsmokers. Am J Epidemiol 152, 1065-1071.

44. Sugiura M, Nakamura M, Ikoma Y et al. (2006) The homeostasis model assessment-insulin resistance index is inversely associated with serum carotenoids in non-diabetic subjects. J Epidemiol 16, 71-78.

45. Juster RP, McEwen BS \& Lupien SJ (2010) Allostatic load biomarkers of chronic stress and impact on health and cognition. Neurosci Biobehav Rev 35, 2-16.

46. Anon. (1994) Plan and operation of the Third National Health and Nutrition Examination Survey, 1988-94. Series 1: programs and collection procedures. Vital Health Stat 1 issue $32,1-407$.

47. Gunter EW, Lewis BG \& Koncikowski SM (1996) Laboratory Procedures used for the Third National Health and Nutrition Examination Survey (NHANES III), 1988-1994. Hyattsville, MD: US Department of Health and Human Services, Centers for Disease Control and Prevention.

48. Centers for Disease Control and Prevention, National Center for Health Statistics (1996) National Health and Nutrition Examination Survey III Examination Data File Documentation. Hyattsville, MD: US Department of Health and Human Services, Centers for Disease Control and Prevention.

49. Centers for Disease Control and Prevention, National Center for Health Statistics (1996) National Health and Nutrition Examination Survey III Laboratory Data File Documentation. Hyattsville, MD: US Department of Health and Human Services, Centers for Disease Control and Prevention.

50. Caraballo RS, Giovino GA, Pechacek TF et al. (2001) Factors associated with discrepancies between self-reports on cigarette smoking and measured serum cotinine levels among persons aged 17 years or older: Third National Health and Nutrition Examination Survey, 1988-1994. Am J Epidemiol 153, 807-814.

51. Ford ES, Zhao G, Tsai J et al. (2011) Low-risk lifestyle behaviors and all-cause mortality: findings from the National Health and Nutrition Examination Survey III Mortality Study. Am J Public Health 101, 1922-1929.

52. Ainsworth BE, Haskell WL, Whitt MC et al. (2000) Compendium of physical activities: an update of activity codes and MET intensities. Med Sci Sports Exerc 32, 9 Suppl., S498-S504.

53. Zhu S, St-Onge MP, Heshka S et al. (2004) Lifestyle behaviors associated with lower risk of having the metabolic syndrome. Metabolism 53, 1503-1511.

54. Mattei J, Noel SE \& Tucker KL (2011) A meat, processed meat, and French fries dietary pattern is associated with high allostatic load in Puerto Rican older adults. J Am Diet Assoc 111, 1498-1506.

55. Da Costa LA, Badawi A \& El-Sohemy A (2012) Nutrigenetics and modulation of oxidative stress. Ann Nutr Metab $\mathbf{6 0}$, Suppl. 3, 27-36.

56. Landrier JF, Marcotorchino J \& Tourniaire F (2012) Lipophilic micronutrients and adipose tissue biology. Nutrients 4, 1622-1649.

57. Calder PC, Albers R, Antoine JM et al. (2009) Inflammatory disease processes and interactions with nutrition. Br J Nutr 101, Suppl. 1, S1-S45.

58. Jonasson L, Wikby A \& Olsson AG (2003) Low serum $\beta$-carotene reflects immune activation in patients with coronary artery disease. Nutr Metab Cardiovasc Dis 13, 120-125.

59. Polidori MC, Cherubini A, Stahl W et al. (2002) Plasma carotenoid and malondialdehyde levels in ischemic stroke patients: relationship to early outcome. Free Radic Res $\mathbf{3 6}$, $265-268$ 
60. Quasim T, McMillan DC, Talwar D et al. (2003) Lower concentrations of carotenoids in the critically ill patient are related to a systemic inflammatory response and increased lipid peroxidation. Clin Nutr 22, 459-462.

61. Kritchevsky SB (1999) $\beta$-Carotene, carotenoids and the prevention of coronary heart disease. J Nutr 129, 5-8.

62. Valtuena S, Del Rio D, Pellegrini N et al. (2007) The total antioxidant capacity of the diet is an independent predictor of plasma $\beta$-carotene. Eur J Clin Nutr 61, 69-76.

63. Kohlmeier L, Kark JD, Gomez-Gracia E et al. (1997) Lycopene and myocardial infarction risk in the EURAMIC Study. Am J Epidemiol 146, 618-626.

64. Peters A \& McEwen BS (2012) Introduction for the allostatic load special issue. Physiol Behav 106, issue 1, 1-4.

65. Tremblay A \& Doucet E (2000) Obesity: a disease or a biological adaptation? Obes Rev 1, 27-35.

66. Block JP, He Y, Zaslavsky AM et al. (2009) Psychosocial stress and change in weight among US adults. Am J Epidemiol 170, 181-192.

67. Pasquali R (2012) The hypothalamic-pituitary-adrenal axis and sex hormones in chronic stress and obesity: pathophysiological and clinical aspects. Ann N Y Acad Sci 1264, 20-35.

68. Vicennati V, Pasqui F, Cavazza C et al. (2009) Stress-related development of obesity and cortisol in women. Obesity (Silver Spring) 17, 1678-1683.

69. Lee JM, Pilli S, Gebremariam A et al. (2010) Getting heavier, younger: trajectories of obesity over the life course. Int $J$ Obes (Lond) 34, 614-623.

70. Chang CY, Chen JY, Ke D et al. (2005) Plasma levels of lipophilic antioxidant vitamins in acute ischemic stroke patients: correlation to inflammation markers and neurological deficits. Nutrition 21, 987-993.
71. Curran FJ, Sattar N, Talwar D et al. (2000) Relationship of carotenoid and vitamins $\mathrm{A}$ and $\mathrm{E}$ with the acute inflammatory response in acute pancreatitis. Br J Surg $\mathbf{8 7}$, 301-305.

72. Beck J, Ferrucci L, Sun K et al. (2008) Circulating oxidized low-density lipoproteins are associated with overweight, obesity, and low serum carotenoids in older communitydwelling women. Nutrition 24, 964-968.

73. Weinbrenner T, Schroder H, Escurriol V et al. (2006) Circulating oxidized LDL is associated with increased waist circumference independent of body mass index in men and women. Am J Clin Nutr 83, 30-35.

74. Gay JL, Salinas JJ, Buchner DM et al. (2013) Meeting physical activity guidelines is associated with lower allostatic load and inflammation in Mexican Americans. $J$ Immigr Minor Health (Epublication ahead of print version).

75. Parente V, Hale L \& Palermo T (2013) Association between breast cancer and allostatic load by race: National Health and Nutrition Examination Survey 1999-2008. Psychooncology 22, 621-628.

76. Gustafsson PE, Janlert U, Theorell $\mathrm{T}$ et al. (2011) Socioeconomic status over the life course and allostatic load in adulthood: results from the Northern Swedish Cohort. J Epidemiol Community Health 65, 986-992.

77. Brady WE, Mares-Perlman JA, Bowen P et al. (1996) Human serum carotenoid concentrations are related to physiologic and lifestyle factors. J Nutr 126, 129-137.

78. Rock CL, Thornquist MD, Kristal AR et al. (1999) Demographic, dietary and lifestyle factors differentially explain variability in serum carotenoids and fat-soluble vitamins: baseline results from the sentinel site of the Olestra PostMarketing Surveillance Study. J Nutr 129, 855-864. 